\title{
Lloyd-Topor Completion and General Stable Models
}

\author{
Vladimir Lifschitz and Fangkai Yang \\ Department of Computer Science \\ The University of Texas at Austin \\ $\{$ vl,fkyang\}@cs.utexas.edu
}

\begin{abstract}
We investigate the relationship between the generalization of program completion defined in 1984 by Lloyd and Topor and the generalization of the stable model semantics introduced recently by Ferraris et al. The main theorem can be used to characterize, in some cases, the general stable models of a logic program by a first-order formula. The proof uses Truszczynski's stable model semantics of infinitary propositional formulas.
\end{abstract}

\section{Introduction}

The theorem by François Fages [1] describing a case when the stable model semantics is equivalent to program completion is one of the most important results in the theory of stable models. It was generalized in [2]3|4], it has led to the invention of loop formulas [5], and it has had a significant impact on the design of answer set solvers.

The general stable model semantics defined in [6] characterizes the stable models of a first-order sentence $F$ as arbitrary models of a certain second-order sentence, denoted by $\mathrm{SM}[F]]_{1}^{1}$ logic programs are viewed there as first-order sentences written in "logic programming notation." In this note we define an extension of Fages' theorem that can be used as a tool for transforming $\mathrm{SM}[F]$, in some cases, into an equivalent first-order formula. That extension refers to the version of program completion introduced by John Lloyd and Rodney Topor in [7]. Their definition allows the body of a rule to contain propositional connectives and quantifiers.

Earlier work in this direction is reported in [6] and [8]. These papers do not mention completion in the sense of Lloyd and Topor explicitly. Instead, they discuss ways to convert a logic program to "Clark normal form" by strongly equivalent transformations [9]10] and completing programs in this normal form by replacing implications with equivalences. But this is essentially what Lloyd-Topor completion does.

The following examples illustrate some of the issues involved. Let $F$ be the program

$$
\begin{aligned}
& p(a), \\
& q(b), \\
& p(x) \leftarrow q(x),
\end{aligned}
$$

\footnotetext{
${ }^{1}$ To be precise, the definition of SM in that paper requires that a set of "intensional predicates" be specified. In the examples below, we assume that all predicate symbols occurring in $F$ are intensional.
} 
or, in other words, the sentence

$$
p(a) \wedge q(b) \wedge \forall x(q(x) \rightarrow p(x)) .
$$

The Clark normal form of (17) is tight in the sense of [6], and Theorem 11 from that paper shows that $\mathrm{SM}[F]$ in this case is equivalent to the conjunction of the completed definitions of $p$ and $q$ :

$$
\begin{aligned}
& \forall x(p(x) \leftrightarrow x=a \vee q(x)), \\
& \forall x(q(x) \leftrightarrow x=b) .
\end{aligned}
$$

Let now $F$ be the program

$$
\begin{aligned}
& p(x) \leftarrow q(x), \\
& q(a) \leftarrow p(b) .
\end{aligned}
$$

This program is not tight in the sense of [6], so that the above-mentioned theorem is not applicable. In fact, $\mathrm{SM}[F]$ is stronger in this case than the conjunction of the completed definitions

$$
\begin{aligned}
& \forall x(p(x) \leftrightarrow q(x)), \\
& \forall x(q(x) \leftrightarrow x=a \wedge p(b)) .
\end{aligned}
$$

A counterexample is provided by any interpretation that treats each of the symbols $p, q$ as a singleton such that its element is equal to both $a$ and $b$. Such a (non-Herbrand) interpretation satisfies (4), but it is not a stable model of (3). (In stable models of (3) both $p$ and $q$ are empty.)

Program (3) is, however, atomic-tight in the sense of [8, Section 5.1.1]. Corollary 5 from that paper allows us to conclude that the equivalence between $\mathrm{SM}[F]$ and $(4)$ is entailed by the unique name assumption $a \neq b$. It follows that the result of applying SM to the program obtained from (4) by adding the constraint

$$
\leftarrow a=b
$$

is equivalent to the conjunction of the completion sentences (4) with $a \neq b$. This example illustrates the role of a property more general than the logical equivalence between $\mathrm{SM}[F]$ and the completion of $F$ : it may be useful to know when the equivalence between these two formulas is entailed by a certain set of assumptions. This information may be relevant if we are interested in a logic program obtained from $F$ by adding constraints.

The result of applying SM to the program

$$
\begin{aligned}
p(a) & \leftarrow p(b), \\
q(c) & \leftarrow q(d), \\
& \leftarrow a=b, \\
& \leftarrow c=d
\end{aligned}
$$

is equivalent to the conjunction of the formulas

$$
\begin{aligned}
\forall x(p(x) \leftrightarrow x=a \wedge p(b)), \\
\forall x(q(x) \leftrightarrow x=c \wedge q(d)), \\
a \neq b, \\
c \neq d .
\end{aligned}
$$


This claim cannot be justified, however, by a reference to Corollary 5 from [8]. The program in this example is atomic-tight, but it does not contain constraints corresponding to some of the unique name axioms, for instance $a \neq c$. We will show how our claim follows from the main theorem stated below.

We will discuss also an example illustrating limitations of earlier work that is related to describing dynamic domains in answer set programming (ASP). The program in that example is not atomic-tight because of rules expressing the commonsense law of inertia. We will show nevertheless that the process of completion can be used to characterize its stable models by a first-order formula.

The class of tight programs is defined in [6] in terms of predicate dependency graphs; that definition is reproduced in Section 3 below. The definition of an atomictight program in [8] refers to more informative "first-order dependency graphs." Our approach is based on an alternative solution to the problem of making predicate dependency graphs more informative, "rule dependency graphs."

After reviewing some background material in Sections 2 and 3 , we define rule dependency graphs in Section 4, state the main theorem and give examples of its use in Sections 5 and 6 and outline its proof in Sections 7 and 8

\section{Review: Operator SM, Lloyd-Topor Programs, and Completion}

In this paper, a formula is a first-order formula that may contain the propositional connectives $\perp$ (logical falsity), $\wedge, \vee$, and $\rightarrow$, and the quantifiers $\forall, \exists$. We treat $\neg F$ as an abbreviation for $F \rightarrow \perp$; † stands for $\perp \rightarrow \perp ; F \leftrightarrow G$ stands for $(F \rightarrow G) \wedge(G \rightarrow F)$.

For any first-order sentence $F$ and any tuple $\mathbf{p}$ of distinct predicate constants (other than equality) $\mathrm{SM}_{\mathbf{p}}[F]$ is the conjunction of $F$ with a second-order "stability condition"; see [6, Section 2] for details. The members of $\mathbf{p}$ are called intensional, and the other predicate constants are extensional. We will drop the subscript in the symbol $\mathrm{SM}_{\mathbf{p}}$ when $\mathbf{p}$ is the list of all predicate symbols occurring in $F$. For any sentence $F$, a p-stable (or simply stable) model of $F$ is an interpretation of the underlying signature that satisfies $\operatorname{SM}_{\mathbf{p}}[F]$.

A Lloyd-Topor program is a finite set of rules of the form

$$
p(\mathbf{t}) \leftarrow G,
$$

where $\mathbf{t}$ is a tuple of terms, and $G$ is a formula. We will identify a program with the sentence obtained by conjoining the formulas

$$
\widetilde{\forall}(G \rightarrow p(\mathbf{t}))
$$

for all its rules 77 . $(\widetilde{\forall} F$ stands for the universal closure of $F$.)

Let $\Pi$ be a Lloyd-Topor program, and $p$ a predicate constant (other than equality). Let

$$
p\left(\mathbf{t}^{i}\right) \leftarrow G^{i} \quad(i=1,2, \ldots)
$$

be all rules of $\Pi$ that contain $p$ in the head. The definition of $p$ in $\Pi$ is the rule

$$
p(\mathbf{x}) \leftarrow \bigvee_{i} \exists \mathbf{y}^{i}\left(\mathbf{x}=\mathbf{t}^{i} \wedge G^{i}\right),
$$


where $\mathbf{x}$ is a list of distinct variables not appearing in any of the rules $(8)$, and $\mathbf{y}^{i}$ is the list of free variables of $(8) 2$ The completed definition of $p$ in $\Pi$ is the formula

$$
\forall \mathbf{x}\left(p(\mathbf{x}) \leftrightarrow \bigvee_{i} \exists \mathbf{y}^{i}\left(\mathbf{x}=\mathbf{t}^{i} \wedge G^{i}\right)\right)
$$

For instance, the completed definitions of $p$ and $q$ in program (1) are the formulas

$$
\begin{aligned}
& \forall x_{1}\left(p\left(x_{1}\right) \leftrightarrow x_{1}=a \vee \exists x\left(x_{1}=x \wedge q(x)\right)\right), \\
& \forall x_{1}\left(q\left(x_{1}\right) \leftrightarrow x_{1}=b\right),
\end{aligned}
$$

which can be equivalently rewritten as (2).

By Comp $[\Pi]$ we denote the conjunction of the completed definitions of all predicate constants $p$ in $\Pi$. This sentence is similar to the completion of $\Pi$ in the sense of [7, Section 2], except that it does not include Clark equality axioms.

\section{Review: Tight Programs}

We will review now the definition of tightness from [6, Section 7.3]. In application to a Lloyd-Topor program $\Pi$, when all predicate constants occurring in $\Pi$ are treated as intensional, that definition can be stated as follows.

An occurrence of an expression in a first-order formula is negated if it belongs to a subformula of the form $\neg F$ (that is, $F \rightarrow \perp$ ), and nonnegated otherwise. The predicate dependency graph of $\Pi$ is the directed graph that has

- all predicate constants occurring in $\Pi$ as its vertices, and

- an edge from $p$ to $q$ whenever $\Pi$ contains a rule (7) with $p$ in the head such that its body $G$ has a positive ${ }^{3}$ nonnegated occurrence of $q$.

We say that $\Pi$ is tight if the predicate dependency graph of $\Pi$ is acyclic.

For example, the predicate dependency graph of program (1) has a single edge, from $p$ to $q$. The predicate dependency graph of program (3) has two edges, from $p$ to $q$ and from $q$ to $p$. The predicate dependency graph of the program

$$
\begin{aligned}
& p(a, b) \\
& q(x, y) \leftarrow p(y, x) \wedge \neg p(x, y)
\end{aligned}
$$

has a single edge, from $q$ to $p$ (because one of the occurrences of $p$ in the body of the second rule is nonnegated). The predicate dependency graph of the program

$$
\begin{aligned}
& p(x) \leftarrow q(x), \\
& q(x) \leftarrow r(x), \\
& r(x) \leftarrow s(x)
\end{aligned}
$$

\footnotetext{
${ }^{2}$ By $\mathbf{x}=\mathbf{t}^{i}$ we denote the conjunction of the equalities between members of the tuple $\mathbf{x}$ and the corresponding members of the tuple $\mathbf{t}^{i}$.

${ }^{3}$ Recall that an occurrence of an expression in a first-order formula is called positive if the number of implications containing that occurrence in the antecedent is even.
} 
has 3 edges:

$$
p \longrightarrow q \longrightarrow r \longrightarrow s
$$

Programs (1), (11) and (12) are tight; program (3) is not.

Proposition 1. If a Lloyd-Topor program $\Pi$ is tight then $\mathrm{SM}[\Pi]$ is equivalent to $\operatorname{Comp}[\Pi]$.

This is an easy corollary to the theorem from [6] mentioned in the introduction. Indeed, consider the set $\Pi^{\prime}$ of the definitions $[9$ of all predicate constants $p$ in $\Pi$. It can be viewed as a formula in Clark normal form in the sense of [6, Section 6.1]. It is tight, because it has the same predicate dependency graph as $\Pi$. By Theorem 11 from [6], $\mathrm{SM}\left[\Pi^{\prime}\right]$ is equivalent to the completion of $\Pi^{\prime}$ in the sense of [6, Section 6.1], which is identical to Comp $[\Pi]$. It remains to observe that $\Pi$ is intuitionistically equivalent to $\Pi^{\prime}$, so that $\mathrm{SM}[\Pi]$ is equivalent to $\mathrm{SM}\left[\Pi^{\prime}\right][6$, Section 5.1].

\section{Rule Dependency Graph}

We are interested in conditions on a Lloyd-Topor program $\Pi$ ensuring that the equivalence

$$
\mathrm{SM}[\Pi] \leftrightarrow \operatorname{Comp}[\Pi]
$$

is entailed by a given set of assumptions $\Gamma$. Proposition 1 gives a solution for the special case when $\Gamma$ is empty. The following definition will help us answer the more general question.

The rule dependency graph of a Lloyd-Topor program $\Pi$ is the directed graph that has

- rules of $\Pi$, with variables (both free and bound) renamed arbitrarily, as its vertices, and

- an edge from a rule $p(\mathbf{t}) \leftarrow G$ to a rule $p^{\prime}\left(\mathbf{t}^{\prime}\right) \leftarrow G^{\prime}$, labeled by an atomic formula $p^{\prime}(\mathbf{s})$, if $p^{\prime}(\mathbf{s})$ has a positive nonnegated occurrence in $G$.

Unlike the predicate dependency graph, the rule dependency graph of a program is usually infinite. For example, the rule dependency graph of program (11) has the vertices $p(a, b)$ and

$$
q\left(x_{1}, y_{1}\right) \leftarrow p\left(y_{1}, x_{1}\right) \wedge \neg p\left(x_{1}, y_{1}\right)
$$

for arbitrary pairs of distinct variables $x_{1}, y_{1}$. It has an edge from each vertex (13) to $p(a, b)$, labeled $p\left(y_{1}, x_{1}\right)$. The rule dependency graph of program 12 has edges of two kinds:

- from $p\left(x_{1}\right) \leftarrow q\left(x_{1}\right)$ to $q\left(x_{2}\right) \leftarrow r\left(x_{2}\right)$, labeled $q\left(x_{1}\right)$, and

- from $q\left(x_{1}\right) \leftarrow r\left(x_{1}\right)$ to $r\left(x_{2}\right) \leftarrow s\left(x_{2}\right)$, labeled $r\left(x_{1}\right)$

for arbitrary variables $x_{1}, x_{2}$.

The rule dependency graph of a program is "dual" to its predicate dependency graph, in the following sense. The vertices of the predicate dependency graph are predicate symbols, and the presence of an edge from $p$ to $q$ is determined by the existence 
of a rule that contains certain occurrences of $p$ and $q$. The vertices of the rule dependency graph are rules, and the presence of an edge from $R_{1}$ to $R_{2}$ is determined by the existence of a predicate symbol with certain occurrences in $R_{1}$ and $R_{2}$.

There is a simple characterization of tightness in terms of rule dependency graphs:

Proposition 2. A Lloyd-Topor program $\Pi$ is tight iff there exists $n$ such that the rule dependency graph of $\Pi$ has no paths of length $n$.

Proof. Assume that $\Pi$ is tight, and let $n$ be the number of predicate symbols occurring in $\Pi$. Then the rule dependency graph of $\Pi$ has no paths of length $n+1$. Indeed, assume that such a path exists:

$$
R_{0} \stackrel{p_{1}(\ldots)}{\longrightarrow} R_{1} \stackrel{p_{2}(\ldots)}{\longrightarrow} R_{2} \stackrel{p_{3}(\ldots)}{\longrightarrow} \ldots \stackrel{p_{n+1}(\ldots)}{\longrightarrow} R_{n+1} .
$$

Each of the rules $R_{i}(1 \leq i \leq n)$ contains $p_{i}$ in the head and a positive nonnegated occurrence of $p_{i+1}$ in the body. Consequently the predicate dependency graph of $\Pi$ has an edge from $p_{i}$ to $p_{i+1}$, so that $p_{1}, \ldots, p_{n+1}$ is a path in that graph; contradiction. Now assume that $\Pi$ is not tight. Then there is an infinite path $p_{1}, p_{2}, \ldots$ in the predicate dependency graph of $\Pi$. Let $R_{i}$ be a rule of $\Pi$ that has $p_{i}$ in the head and a positive nonnegated occurrence of $p_{i+1}$ in the body. Then the rule dependency graph of $\Pi$ has an infinite path of the form

$$
R_{1} \stackrel{p_{2}(\ldots)}{\longrightarrow} R_{2} \stackrel{p_{3}(\ldots)}{\longrightarrow} \ldots
$$

The main theorem, stated in the next section, refers to finite paths in the rule dependency graph of a program $\Pi$ that satisfy an additional condition: the rules at their vertices have no common variables (neither free nor bound). Such paths will be called chains.

Corollary 1. A Lloyd-Topor program $\Pi$ is tight iff there exists $n$ such that $\Pi$ has no chains of length $n$.

Indeed, any finite path in the rule dependency graph of $\Pi$ can be converted into a chain of the same length by renaming variables.

\section{Main Theorem}

Let $C$ be a chain

$$
\begin{gathered}
p_{0}\left(\mathbf{t}^{0}\right) \leftarrow{ }_{1} \text { Body }_{0} \\
\downarrow p_{1}\left(\mathbf{s}^{1}\right) \\
p_{1}\left(\mathbf{t}^{1}\right) \leftarrow 1 B o d y_{1} \\
\downarrow p_{2}\left(\mathbf{s}^{2}\right) \\
\ldots \ldots \ldots \ldots \\
\downarrow p_{n}\left(\mathbf{s}^{n}\right) \\
p_{n}\left(\mathbf{t}^{n}\right) \leftarrow 1 B o d y_{n}
\end{gathered}
$$


in a Lloyd-Topor program $\Pi$. The corresponding chain formula $F_{C}$ is the conjunction

$$
\bigwedge_{i=1}^{n} \mathbf{s}^{i}=\mathbf{t}^{i} \wedge \bigwedge_{i=0}^{n} 1 B^{B} d y_{i}
$$

For instance, if $C$ is the chain

$$
\begin{gathered}
q\left(x_{1}, y_{1}\right) \leftarrow p\left(y_{1}, x_{1}\right) \wedge \neg p\left(x_{1}, y_{1}\right) \\
\downarrow \downarrow p\left(y_{1}, x_{1}\right) \\
p(a, b)
\end{gathered}
$$

in program 11 then $F_{C}$ is

$$
y_{1}=a \wedge x_{1}=b \wedge p\left(y_{1}, x_{1}\right) \wedge \neg p\left(x_{1}, y_{1}\right) .
$$

Let $\Gamma$ be a set of sentences. About a Lloyd-Topor program $\Pi$ we will say that it is tight relative to $\Gamma$, or $\Gamma$-tight, if there exists a positive integer $n$ such that, for every chain $C$ in $\Pi$ of length $n$,

$$
\Gamma, \operatorname{Comp}[\Pi] \models \widetilde{\forall} \neg F_{C} .
$$

Main Theorem. If a Lloyd-Topor program $\Pi$ is $\Gamma$-tight then

$$
\Gamma \models \operatorname{SM}[\Pi] \leftrightarrow \operatorname{Comp}[\Pi] .
$$

Corollary 1 shows that every tight program is trivially $\Gamma$-tight even when $\Gamma$ is empty. Consequently the main theorem can be viewed as a generalization of Proposition 1.

Tightness in the sense of Section 3 is a syntactic condition that is easy to verify; $\Gamma$-tightness is not. Nevertheless, the main theorem is useful because it may allow us to reduce the problem of characterizing the stable models of a program by a first-order formula to verifying an entailment in first-order logic.

Here are some examples. In each case, to verify $\Gamma$-tightness we take $n=1$. We will check the entailment in the definition of $\Gamma$-tightness by deriving a contradiction from (some subset of) the assumptions $\Gamma$, $\operatorname{Comp}[\Pi]$, and $F_{C}$.

Example 1. The one-rule program

$$
p(a) \leftarrow p(x) \wedge x \neq a
$$

is tight relative to $\emptyset$. Indeed, any chain of length 1 has the form

$$
\begin{gathered}
p(a) \leftarrow p\left(x_{1}\right) \wedge x_{1} \neq a \\
\quad \downarrow d p\left(x_{1}\right) \\
p(a) \leftarrow p\left(x_{2}\right) \wedge x_{2} \neq a .
\end{gathered}
$$

The corresponding chain formula

$$
x_{1}=a \wedge p\left(x_{1}\right) \wedge x_{1} \neq a \wedge p\left(x_{2}\right) \wedge x_{2} \neq a .
$$


is contradictory.

Thus the stable models of this program are described by its completion, even though the program is not tight (and not even atomic-tight).

Example 2. Let $\Pi$ be the program consisting of the first 2 rules of (5):

$$
\begin{aligned}
& p(a) \leftarrow p(b), \\
& q(c) \leftarrow q(d) .
\end{aligned}
$$

To justify the claim about (5) made in the introduction, we will check that $\Pi$ is tight relative to $\{a \neq b, c \neq d\}$. There are two chains of length 1 :

$$
\begin{gathered}
p(a) \leftarrow p(b) \\
\downarrow p(b) \\
p(a) \leftarrow p(b)
\end{gathered}
$$

and

$$
\begin{gathered}
q(c) \leftarrow q(d) \\
\downarrow q(d) \\
q(c) \leftarrow q(d) .
\end{gathered}
$$

The corresponding chain formulas are

$$
b=a \wedge p(b) \wedge p(b)
$$

and

$$
d=c \wedge q(d) \wedge q(d)
$$

Each of them contradicts $\Gamma$.

Example 3. Let us check that program $(3)$ is tight relative to $\{a \neq b\}$. Its chains of length 1 are

$$
\begin{gathered}
p\left(x_{1}\right) \leftarrow q\left(x_{1}\right) \\
\downarrow \downarrow \\
q\left(x_{1}\right) \\
q(a) \leftarrow p(b)
\end{gathered}
$$

and

$$
\begin{gathered}
q(a) \leftarrow p(b) \\
\downarrow \downarrow \\
p(b) \\
p\left(x_{1}\right) \leftarrow q\left(x_{1}\right)
\end{gathered}
$$

for an arbitrary variable $x_{1}$. The corresponding chain formulas include the conjunctive term $p(b)$. Using the completion 40 of the program, we derive $b=a$, which contradicts $\Gamma$.

\section{A Larger Example}

Programs found in actual applications of ASP usually involve constructs that are not allowed in Lloyd-Topor programs, such as choice rules and constraints. Choice rules have the form

$$
\{p(\mathbf{t})\} \leftarrow G .
$$


We view this expression as shorthand for the sentence

$$
\widetilde{\forall}(G \rightarrow p(\mathbf{t}) \vee \neg p(\mathbf{t})) .
$$

A constraint $\leftarrow G$ is shorthand for the sentence $\widetilde{\forall} \neg G$. Such sentences do not correspond to any rules in the sense of Section 2

Nevertheless, the main theorem stated above can sometimes help us characterize the stable models of a "realistic" program by a first-order formula. In this section we discuss an example of this kind.

The logic program $M$ described below encodes commonsense knowledge about moving objects from one location to another. Its signature consists of

- the object constants $\widehat{0}, \ldots, \widehat{k}$, where $k$ is a fixed nonnegative integer;

- the unary predicate constants iobject, iplace, and istep; they correspond to the three types of individuals under consideration;

- the binary predicate constant inext; it describes the temporal order of steps;

- the ternary predicate constants iat and imove; they represent the fluents and actions that we are interested in.

The predicate constants 1step, 1next, and 1at are intensional; the other three are not. (The fact that some predicates are extensional is the first sign that $M$ is not a Lloyd-Topor program.) The program consists of the following rules:

(i) the facts

$$
\begin{aligned}
& 1 \operatorname{step}(\widehat{0}), 1 \operatorname{step}(\widehat{1}), \ldots 1 \operatorname{step}(\widehat{k}) ; \\
& \operatorname{1next}(\widehat{0}, \widehat{1}), \operatorname{1next}(\widehat{1}, \widehat{2}), \ldots, \operatorname{1next}(\widehat{k-1}, \widehat{k})
\end{aligned}
$$

(ii) the unique name constraints

$$
\leftarrow \widehat{i}=\widehat{j} \quad(1 \leq i<j \leq k)
$$

(iii) the constraints describing the arguments of rat and imove:

$$
\leftarrow \operatorname{1at}(x, y, z) \wedge \neg(\operatorname{1object}(x) \wedge \operatorname{splace}(y) \wedge 1 \operatorname{step}(z))
$$

and

$$
\leftarrow \operatorname{imove}(x, y, z) \wedge \neg(\operatorname{1object}(x) \wedge \operatorname{iplace}(y) \wedge 1 \operatorname{step}(z)) ;
$$

(iv) the uniqueness of location constraint

$$
\leftarrow \operatorname{lat}\left(x, y_{1}, z\right) \wedge \mathfrak{1 a t}\left(x, y_{2}, z\right) \wedge y_{1} \neq y_{2} ;
$$

(v) the existence of location constraint

$$
\leftarrow \operatorname{robject}(x) \wedge 1 \operatorname{step}(z) \wedge \neg \exists y \operatorname{1at}(x, y, z) ;
$$

(vi) the rule expressing the effect of moving an object:

$$
\operatorname{lat}(x, y, u) \leftarrow \operatorname{imove}(x, y, z) \wedge \operatorname{1next}(z, u) ;
$$


(vii) the choice rule expressing that initially an object can be anywhere:

$$
\{\operatorname{lat}(x, y, 0)\} \leftarrow \operatorname{robject}(x) \wedge \operatorname{pplace}(y) ;
$$

(viii) the choice rule expressing the commonsense law of inertia. ${ }^{4}$

$$
\{\operatorname{lat}(x, y, u)\} \leftarrow \operatorname{lat}(x, y, z) \wedge \operatorname{inext}(z, u) .
$$

Program $M$ is not atomic-tight, so that methods of [8] are not directly applicable to it. Nevertheless, we can describe the stable models of this program without the use of second-order quantifiers. In the statement of the proposition below, $\mathbf{p}$ stands for the list of intensional predicates 1step, inext and $1 a t$, and $H$ is the conjunction of the universal closures of the formulas

$$
\begin{aligned}
& \widehat{i} \neq \widehat{j} \quad(1 \leq i<j \leq k), \\
& 1 \operatorname{at}(x, y, z) \rightarrow \operatorname{lobject}(x) \wedge \operatorname{1place}(y) \wedge \operatorname{step}(z), \\
& \operatorname{1move}(x, y, z) \rightarrow \operatorname{1object}(x) \wedge \operatorname{1place}(y) \wedge \operatorname{step}(z), \\
& \operatorname{1at}\left(x, y_{1}, z\right) \wedge \operatorname{1at}\left(x, y_{2}, z\right) \rightarrow y_{1}=y_{2}, \\
& \operatorname{lobject}(x) \wedge \operatorname{step}(z) \rightarrow \exists y \operatorname{1at}(x, y, z) .
\end{aligned}
$$

Proposition 3. $\mathrm{SM}_{\mathbf{p}}[M]$ is equivalent to the conjunction of $H$ with the universal closures of the formulas

$$
\begin{aligned}
& 1 \operatorname{step}(z) \leftrightarrow \bigvee_{i=0}^{k} z=\widehat{i} \\
& \operatorname{lnext}(z, u) \leftrightarrow \bigvee_{i=0}^{k-1}(z=\widehat{i} \wedge u=\widehat{i+1}) \\
& \operatorname{lat}(x, y, \widehat{i+1}) \leftrightarrow(\operatorname{move}(x, y, \widehat{i}) \vee(\operatorname{lat}(x, y, \widehat{i}) \wedge \neg \exists w \operatorname{move}(x, w, \hat{i}))) \\
& (i=0, \ldots, k-1) .
\end{aligned}
$$

Recall that the effect of adding a constraint to a logic program is to eliminate its stable models that violate that constraint [6, Theorem 3]. An interpretation satisfies $H$ iff it does not violate any of the constraints (ii)-(v). So the statement of Proposition 3 can be summarized as follows: the contribution of rules (i) and (vi)-(viii), under the stable model semantics, amounts to providing explicit definitions for istep and inext, and "successor state formulas" for at.

The proof of Proposition 3 refers to the Lloyd-Topor program $\Pi$ consisting of rules (i), (vi),

(vii') $\operatorname{lat}(x, y, 0) \leftarrow \operatorname{lobject}(x) \wedge \operatorname{pplace}(y) \wedge \neg \neg 1$ at $(x, y, 0)$,

(viii') 1 at $(x, y, u) \leftarrow \operatorname{lat}(x, y, z) \wedge \operatorname{lnext}(z, u) \wedge \neg \neg 1$ at $\left(x, y, t_{2}\right)$,

and

$$
\begin{aligned}
\operatorname{lobject}(x) & \leftarrow \neg \neg \text { lobject }(x), \\
\operatorname{lplace}(y) & \leftarrow \neg \neg \text { place }(y), \\
\text { 1move }(x, y, z) & \leftarrow \neg \neg \text { move }(x, y, z) .
\end{aligned}
$$

\footnotetext{
${ }^{4}$ This representation of inertia follows the example of [?, Figure 1].
} 
It is easy to see that $\mathrm{SM}_{\mathbf{p}}[M]$ is equivalent to $\mathrm{SM}[\Pi] \wedge H$. Indeed, consider the program $M^{\prime}$ obtained from $M$ by adding rules 18 . These rules are strongly equivalent to the choice rules

$$
\{\operatorname{lobject}(x)\},\{\operatorname{lplace}(y)\},\{\operatorname{lmove}(x, y, z)\} .
$$

Consequently $\mathrm{SM}_{\mathbf{p}}[M]$ is equivalent to $\mathrm{SM}\left[M^{\prime}\right]$ [6, Theorem 2]. It remains to notice that (vii) is strongly equivalent to (vii'), and (viii) is strongly equivalent to (viii').

Furthermore - and this is the key step in the proof of Proposition 3 - the secondorder formula $\mathrm{SM}[\Pi] \wedge H$ is equivalent to the first-order formula $\operatorname{Comp}[\Pi] \wedge H$, in view of our main theorem and the following fact:

\section{Lemma 1. Program II is H-tight.}

To derive Proposition 3 from the lemma, we only need to observe that (15) and (16) are the completed definitions of istep and inext in $\Pi$, and that the completed definition of lat can be transformed into (17) under assumptions [15, , 16, and $H$.

Proof of Lemma 1 . Consider a chain in $\Pi$ of length $k+2$ :

$$
R_{0} \stackrel{p_{1}(\ldots)}{\longrightarrow} R_{1} \stackrel{p_{2}(\ldots)}{\longrightarrow} \ldots \stackrel{p_{k+1}(\ldots)}{\longrightarrow} R_{k+1} \stackrel{p_{k+2}(\ldots)}{\longrightarrow} R_{k+2} .
$$

Each $R_{i}$ is obtained from one of the rules (i), (vi), (vii'), (viii'), 18p by renaming variables. Each $p_{i}$ occurs in the head of $R_{i}$ and has a positive nonnegated occurrence in $R_{i-1}$. Since there are no nonnegated predicate symbols in the bodies of rules (i) and (18), we conclude that $R_{0}, \ldots, R_{k+1}$ are obtained from other rules of $\Pi$, that is, from (vi), (vii'), and (viii'). Since the predicate constant in the head of each of these three rules is 1at, each of $p_{1}, \ldots, p_{k+1}$ is the symbol at. Since there are no nonnegated occurrences of at in the bodies of (vi) and (vii'), we conclude that $R_{0}, \ldots, R_{k}$ are obtained by renaming variables in (viii'). This means that chain 18 has the form

$$
\begin{aligned}
& \operatorname{lat}\left(x_{0}, y_{0}, u_{0}\right) \leftarrow \operatorname{lat}\left(x_{0}, y_{0}, z_{0}\right) \wedge \operatorname{inext}\left(z_{0}, u_{0}\right) \wedge \neg \neg \operatorname{lat}\left(x_{0}, y_{0}, u_{0}\right) \\
& \downarrow \text { iat }\left(x_{0}, y_{0}, z_{0}\right) \\
& \operatorname{lat}\left(x_{1}, y_{1}, u_{1}\right) \leftarrow \operatorname{lat}\left(x_{1}, y_{1}, z_{1}\right) \wedge \operatorname{lnext}\left(z_{1}, u_{1}\right) \wedge \neg \neg 1 \operatorname{at}\left(x_{1}, y_{1}, u_{1}\right) \\
& \downarrow_{1} a t\left(x_{1}, y_{1}, z_{1}\right) \\
& \text { i. } \\
& \downarrow 1 a t\left(x_{k-1}, y_{k-1}, z_{k-1}\right) \\
& \operatorname{lat}\left(x_{k}, y_{k}, u_{k}\right) \leftarrow \operatorname{lat}\left(x_{k}, y_{k}, z_{k}\right) \wedge \operatorname{lnext}\left(z_{k}, u_{k}\right) \wedge \neg \neg \operatorname{at}\left(x_{k}, y_{k}, u_{k}\right) \\
& \downarrow_{1} a t\left(x_{k}, y_{k}, z_{k}\right) \\
& R_{k+1} \\
& \downarrow \cdots \\
& R_{k+2} \text {. }
\end{aligned}
$$

The corresponding chain formula contains the conjunctive terms

$$
z_{0}=u_{1}, z_{1}=u_{2}, \ldots, z_{k-1}=u_{k}
$$


and

$$
\operatorname{lnext}\left(z_{0}, u_{0}\right), \operatorname{1next}\left(z_{1}, u_{1}\right), \ldots, \operatorname{lnext}\left(z_{k}, u_{k}\right) .
$$

From these formulas we derive

$$
\operatorname{inext}\left(u_{1}, u_{0}\right), \operatorname{inext}\left(u_{2}, u_{1}\right), \ldots, \operatorname{inext}\left(u_{k+1}, u_{k}\right),
$$

where $u_{k+1}$ stands for $z_{k}$. Using the completed definition of inext, we conclude:

$$
u_{i}=\widehat{0} \vee \cdots \vee u_{i}=\widehat{k} \quad(0 \leq i \leq k+1) .
$$

Consider the case when

$$
u_{i}=\widehat{j_{i}} \quad(0 \leq i \leq k+1)
$$

for some numbers $j_{0}, \ldots, j_{k+1} \in\{0, \ldots, k\}$. There exists at least one subscript $i$ such that $j_{i} \neq j_{i+1}+1$, because otherwise we would have

$$
j_{0}=j_{1}+1=j_{2}+2=\cdots=j_{k+1}+k+1,
$$

which is impossible because $j_{0}, j_{k+1} \in\{0, \ldots, k\}$. By the choice of $i$, from the completed definition of unext and the unique name assumption (included in $H$ ) we can derive $\neg 1$ ext $\left(\widehat{j_{i+1}}, \widehat{j_{i}}\right)$. Consequently $\neg 1$ next $\left(u_{i+1}, u_{i}\right)$, which contradicts 20 .

\section{Review: Stable Models of Infinitary Formulas}

Our proof of the main theorem employs the method proposed (for a different purpose) by Miroslaw Truszczynski [11], and in this section we review some of the definitions and results of that paper. The stable model semantics of propositional formulas due to Paolo Ferraris [12] is extended there to formulas with infinitely long conjunctions and disjunctions, and that generalization is related to the operator SM.

Let $\mathcal{A}$ be a set of propositional atoms. The sets $\mathcal{F}_{0}, \mathcal{F}_{1}, \ldots$ are defined as follows:

- $\mathcal{F}_{0}=\mathcal{A} \cup\{\perp\}$

- $\mathcal{F}_{i+1}$ consists of expressions $\mathcal{H}^{\vee}$ and $\mathcal{H}^{\wedge}$, for all subsets $\mathcal{H}$ of $\mathcal{F}_{0} \cup \ldots \cup \mathcal{F}_{i}$, and of expressions $F \rightarrow G$, where $F, G \in \mathcal{F}_{0} \cup \ldots \cup \mathcal{F}_{i}$.

An infinitary formula (over $\mathcal{A}$ ) is an element of $\bigcup_{i=0}^{\infty} \mathcal{F}_{i}$.

A (propositional) interpretation is a subset of $\mathcal{A}$. The satisfaction relation between an interpretation and an infinitary formula is defined in a natural way. The definition of the reduct $F^{I}$ of a formula $F$ relative to an interpretation $I$ proposed in [12] is extended to infinitary formulas as follows:

$-\perp^{I}=\perp$.

- For $A \in \mathcal{A}, A^{I}=\perp$ if $I \not \models A$; otherwise $A^{I}=A$.

- $\left(\mathcal{H}^{\wedge}\right)^{I}=\perp$ if $I \not \models \mathcal{H}^{\wedge}$; otherwise $\left(\mathcal{H}^{\wedge}\right)^{I}=\left\{G^{I} \mid G \in \mathcal{H}\right\}^{\wedge}$.

- $\left(\mathcal{H}^{\vee}\right)^{I}=\perp$ if $I \not \models \mathcal{H}^{\vee}$; otherwise $\left(\mathcal{H}^{\vee}\right)^{I}=\left\{G^{I} \mid G \in \mathcal{H}\right\}^{\vee}$.

- $(G \rightarrow H)^{I}=\perp$ if $I \not \models G \rightarrow H$; otherwise $(G \rightarrow H)^{I}=G^{I} \rightarrow H^{I}$. 
An interpretation $I$ is a stable model of an infinitary formula $F$ if $I$ is a minimal model of $F^{I}$. An interpretation $I$ satisfies $F^{I}$ iff it satisfies $F$ [11, Proposition 1], so that stable models of $F$ are models of $F$.

Infinitary formulas are used to encode first-order sentences as follows. For any interpretation $I$ in the sense of first-order logic, let $\mathcal{A}$ be the set of ground atoms formed from the predicate constants of the underlying signature and the "names" $\xi^{*}$ of elements $\xi$ of the universe $|I|$ of $I$-new objects constants that are in a 1-1 correspondence with elements of $|I|$. By $I^{r}$ we denote the set of atoms from $\mathcal{A}$ that are satisfied by $I$. In the definition below, $t^{I}$ stands for the value assigned to the ground term $t$ by the interpretation $I$. The grounding of a first-order sentence $F$ relative to $I$ (symbolically, $\lg _{I}(F)$ ) is the infinitary formula over $A$ constructed as follows:

- $\operatorname{1gr}_{I}(\perp)=\perp$.

$-\operatorname{lgr}_{I}\left(p\left(t_{1}, \ldots, t_{k}\right)\right)=p\left(\left(t_{1}^{I}\right)^{*}, \ldots,\left(t_{k}^{I}\right)^{*}\right)$.

- ${ }_{1} g r_{I}\left(t_{1}=t_{2}\right)=\top$, if $t_{1}^{I}=t_{2}^{I}$, and $\perp$ otherwise.

- If $F=G \vee H, 1 g r_{I}(F)=1 g r_{I}(G) \vee 1 g r_{I}(H)$ (the case of $\wedge$ is analogous).

- If $F=G \rightarrow H, 1 g r_{I}(F)=1 g r_{I}(G) \rightarrow 1 g r_{I}(H)$.

- If $F=\exists x G(x), 1 g r_{I}(F)=\left\{1 g r_{I}\left(G\left(u^{*}\right)\right)|u \in| I \mid\right\}^{\vee}$.

- If $F=\forall x G(x), \operatorname{lgr}_{I}(F)=\left\{\operatorname{lgr}_{I}\left(G\left(u^{*}\right)\right)|u \in| I \mid\right\}^{\wedge}$.

It is easy to check that $1 g r_{I}$ is a faithful translation in the following sense: $I$ satisfies a first-order sentence $F$ iff $I^{r}$ satisfies $1 g r_{I}(F)$.

This transformation is also faithful in the sense of the stable model semantics: $I$ satisfies $\mathrm{SM}[F]$ iff $I^{r}$ is a stable model of $\operatorname{lgr}_{I}(F)[11$, Theorem 5]. This is why infinitary formulas can be used for proving properties of the operator SM.

\section{Proof Outline}

In the statement of the main theorem, the implication left-to-right

$$
\mathrm{SM}[\Pi] \rightarrow \operatorname{Comp}[\Pi]
$$

is logically valid for any Lloyd-Topor program $\Pi$. This fact follows from [6, Theorem 11] by the argument used in the proof of Proposition 1 above. In this section we outline the proof in the other direction:

If a Lloyd-Topor program $\Pi$ is $\Gamma$-tight, and an interpretation $I$ satisfies both $\Gamma$ and $\operatorname{Comp}[\Pi]$, then I satisfies $\mathrm{SM}[\Pi]$.

This assertion follows from three lemmas. The first of them expresses a Fagesstyle property of infinitary formulas similar to Theorem 1 from [3]. It deals with infinitary programs - conjunctions of (possibly infinitely many) implications $G \rightarrow A$ with $A \in \mathcal{A}$. Such an implication will be called an (infinitary) rule with the head $A$ and body $G$, and we will write it as $A \leftarrow G$. For instance, if $\Pi$ is a Lloyd-Topor program 
then, for any interpretation $I, 1 g r_{I}(\Pi)$ is an infinitary program. We say that an interpretation $I$ is supported by an infinitary program $\Pi$ if each atom $A \in I$ is the head of a rule $A \leftarrow G$ of $\Pi$ such that $I \models G$. The lemma shows that under some condition the stable models of an infinitary program $\Pi$ can be characterized as the interpretations that satisfy $\Pi$ and are supported by $\Pi$.

The condition refers to the set of positive nonnegated atoms of an infinitary formula. This set, denoted by $\operatorname{Pnn}(F)$, and the set of negative nonnegated atoms of $F$, denoted by $\operatorname{Nnn}(F)$, are defined recursively, as follows:

$-\operatorname{Pnn}(\perp)=\emptyset$.

- For $A \in \mathcal{A}, \operatorname{Pnn}(A)=\{A\}$.

- $\operatorname{Pnn}\left(\mathcal{H}^{\wedge}\right)=\operatorname{Pnn}\left(\mathcal{H}^{\vee}\right)=\bigcup_{H \in \mathcal{H}} \operatorname{Pnn}(H)$.

- $\operatorname{Pnn}(G \rightarrow H)= \begin{cases}\emptyset & \text { if } H=\perp, \\ \operatorname{Nnn}(G) \cup \operatorname{Pnn}(H) & \text { otherwise. }\end{cases}$

$-\operatorname{Nnn}(\perp)=\emptyset$,

- For $A \in \mathcal{A}, \operatorname{Nnn}(A)=\emptyset$.

- $\operatorname{Nnn}\left(\mathcal{H}^{\wedge}\right)=\operatorname{Nnn}\left(\mathcal{H}^{\vee}\right)=\bigcup_{H \in \mathcal{H}} \operatorname{Nnn}(H)$.

- $\operatorname{Nnn}(G \rightarrow H)= \begin{cases}\emptyset & \text { if } H=\perp, \\ \operatorname{Pnn}(G) \cup \operatorname{Nnn}(H) & \text { otherwise. }\end{cases}$

Let $\Pi$ be an infinitary program, and $I$ a propositional interpretation. About atoms $A, A^{\prime} \in I$ we say that $A^{\prime}$ is a parent of $A$ relative to $\Pi$ and $I$ if $\Pi$ has a rule $A \leftarrow G$ with the head $A$ such that $I \models G$ and $A^{\prime}$ is a positive nonnegated atom of $G$. We say that $\Pi$ is tight on $I$ if there is no infinite sequence $A_{0}, A_{1}, \ldots$ of elements of $I$ such that for every $i, A_{i+1}$ is a parent of $A_{i}$ relative to $F$ and $I$.

Lemma 2. For any model I of an infinitary program $\Pi$ such that $\Pi$ is tight on $I, I$ is stable iff $I$ is supported by $\Pi$.

The next lemma relates the $\Gamma$-tightness condition from the statement of the main theorem to tightness on an interpretation defined above.

Lemma 3. If a Lloyd-Topor program $\Pi$ is $\Gamma$-tight, and an interpretation $I$ satisfies both $\Gamma$ and $\operatorname{Comp}[\Pi]$, then $\operatorname{1gr}_{I}(\Pi)$ is tight on $I^{r}$.

Finally, models of Comp $[\Pi]$ can be characterized in terms of satisfaction and supportedness:

Lemma 4. For any Lloyd-Topor program $\Pi$, an interpretation I satisfies $\operatorname{Comp}[\Pi]$ iff $I^{r}$ satisfies $1 g r_{I}(\Pi)$ and is supported by $1 g r_{I}(\Pi)$.

Proofs of Lemmas 24 can be found in the longer version of the paper, posted at http://www.cs.utexas.edu/users/vl/papers/ltc-long.pdf. 


\section{Conclusion}

We proposed a new method for representing $\mathrm{SM}[F]$ in the language of first-order logic. It is more general than the approach of [6]. Its relationship with the ideas of [8] requires further study. This method allows us, in particular, to prove the equivalence of some ASP descriptions of dynamic domains to axiomatizations based on successor state axioms.

The use of the stable model semantics of infinitary formulas [11] in the proof of the main theorem illustrates the potential of that semantics as a tool for the study of the operator SM.

\section{Acknowledgements}

We are grateful to Joohyung Lee and to the anonymous referees for useful comments.

\section{References}

1. Fages, F.: Consistency of Clark's completion and existence of stable models. Journal of Methods of Logic in Computer Science 1 (1994) 51-60

2. Lifschitz, V.: Foundations of logic programming. In Brewka, G., ed.: Principles of Knowledge Representation. CSLI Publications (1996) 69-128

3. Erdem, E., Lifschitz, V.: Tight logic programs. Theory and Practice of Logic Programming 3 (2003) 499-518

4. Lin, F., Zhao, J.: On tight logic programs and yet another translation from normal logic programs to propositional logic. In: Proceedings of International Joint Conference on Artificial Intelligence (IJCAI). (2003) 853-864

5. Lin, F., Zhao, Y.: ASSAT: Computing answer sets of a logic program by SAT solvers. Artificial Intelligence 157 (2004) 115-137

6. Ferraris, P., Lee, J., Lifschitz, V.: Stable models and circumscription. Artificial Intelligence 175 (2011) 236-263

7. Lloyd, J., Topor, R.: Making Prolog more expressive. Journal of Logic Programming 3 (1984) 225-240

8. Lee, J., Meng, Y.: First-order stable model semantics and first-order loop formulas. J. Artif. Intell. Res. (JAIR) 42 (2011) 125-180

9. Lifschitz, V., Pearce, D., Valverde, A.: Strongly equivalent logic programs. ACM Transactions on Computational Logic 2 (2001) 526-541

10. Lifschitz, V., Pearce, D., Valverde, A.: A characterization of strong equivalence for logic programs with variables. In: Procedings of International Conference on Logic Programming and Nonmonotonic Reasoning (LPNMR). (2007)

11. Truszczynski, M.: Connecting first-order asp and the logic fo(id) through reducts. (2012)

12. Ferraris, P.: Answer sets for propositional theories. In: Proceedings of International Conference on Logic Programming and Nonmonotonic Reasoning (LPNMR). (2005) 119-131 